\title{
Takotsubo Cardiomyopathy Post Induction of Anesthesia
}

\author{
Ruth Bird ${ }^{\mathrm{a}, \mathrm{b}}$, Aarash Saleh ${ }^{\mathrm{a}}$, Tom Riley ${ }^{\mathrm{a}}$, Hannah Smitha ${ }^{\mathrm{a}}$, Charlotte Dempsey ${ }^{\mathrm{a}}$
}

\begin{abstract}
We report the case of a 67 -year-old female patient who underwent radiofrequency ablation of a renal cell carcinoma, and developed dynamic ST elevation immediately following induction of anesthesia. She was transferred to the cardiac catheterization laboratory where she underwent a coronary angiogram. This showed no obstruction of the coronary arteries, but an echocardiogram demonstrated an ejection fraction of $20 \%$ with a picture suggestive of Takotsubo cardiomyopathy. The patient later developed pulmonary edema requiring continuous positive pressure ventilation (CPAP), inotropes and vasopressors. She made a good recovery by the third day following induction, with resolution of her elevated ST segments, troponin and impaired ejection fraction.
\end{abstract}

Keywords: Takotsubo cardiomyopathy; Anesthesia; ST elevation

\section{Introduction}

Takotsubo cardiomyopathy, also known as transient left ventricular apical ballooning (TLVAB) or stress cardiomyopathy, was first described in by Sato et al in 1990. Presentation frequently mimics acute myocardial infarction with chest pain and pulmonary edema and electrocardiogram (ECG) changes including QT prolongation and precordial ST elevation with deep T-wave inversion [1]. Cardiac enzymes are typically elevated. The hallmark feature on echocardiography and left ventriculography is balloon-like apical left ventricular akinesia, which resembles a Japanese takotsubo (octopus trap) with its round base and narrow neck [2, 3].

Annual population incidence is estimated at between $0.00006 \%$ and $0.05 \%$ and occurs more frequently in postmenopausal females [4].

Manuscript accepted for publication August 19, 2015

aRoyal London Hospital, Whitechapel Road, Whitechapel, London E1 1BB UK

${ }^{b}$ Corresponding Author: Ruth Bird, Royal London Hospital, Whitechapel Road, Whitechapel, London E1 1BB, UK. Email: ruthHbird@doctors.org.uk

doi: http://dx.doi.org/10.14740/jmc2280w
Onset is frequently triggered by an acute emotional or physiological stress event, prompting the name "broken heart syndrome". Although the exact mechanism of the acute reversible regional wall abnormality remains unknown, it has been suggested that a direct effect of catecholamine on the myocardial surface may be responsible. The apical segment of the left ventricle is trabeculated and thus has a high surface to volume ratio rendering it more exposed to catecholamine toxicity [5].

Takotsubo cardiomyopathy associated with surgery or anesthesia is a well-recognized phenomenon but is generally described as a post-operative deterioration. It has been suggested that the pain associated with emergence from anesthesia and associated anxiety may contribute, and this patient developed this complication at induction.

\section{Case Report}

A 67-year-old female patient was admitted electively for radiofrequency ablation of an incidentally found $2.5 \mathrm{~cm}$ right renal cell carcinoma (radiological grade T1aN0M0). This tumor was diagnosed on a chest/abdominal/pelvic computed tomography (CT) scan during a previous admission for a community acquired respiratory infection.

The patient had a background history of pulmonary fibrosis and chronic obstructive pulmonary disease, managed with inhalers. Her last steroid use was 5 months prior to admission. She was prescribed methotrexate and adalimumab for rheumatoid arthritis. Her pre-admission exercise tolerance was limited by her respiratory pathology to five steps and she was able to manage a flight of stairs, though she would normally stop 2 - 3 times on the way up, again, due to her shortness of breath.

Her lung function was tested 2 weeks prior to admission and showed a peak expiratory flow rate (PEFR) of $250 \mathrm{~L} / \mathrm{min}$, forced expiratory volume in 1 second (FEV1) of $1.60 \mathrm{~L}$ with a total lung capacity (TLC) of 4.03. Her significant cardiac risk factors were her age, previous smoking history and high cholesterol, which was well controlled on a statin.

Her pre-operative ECG showed a sinus tachycardia at a rate of 100 with poor R-wave progression and she was anxious prior to the procedure. The patient had undergone two previous general anesthetics without complications, the latest being 2 years prior to her admission. Since this she has had no further 


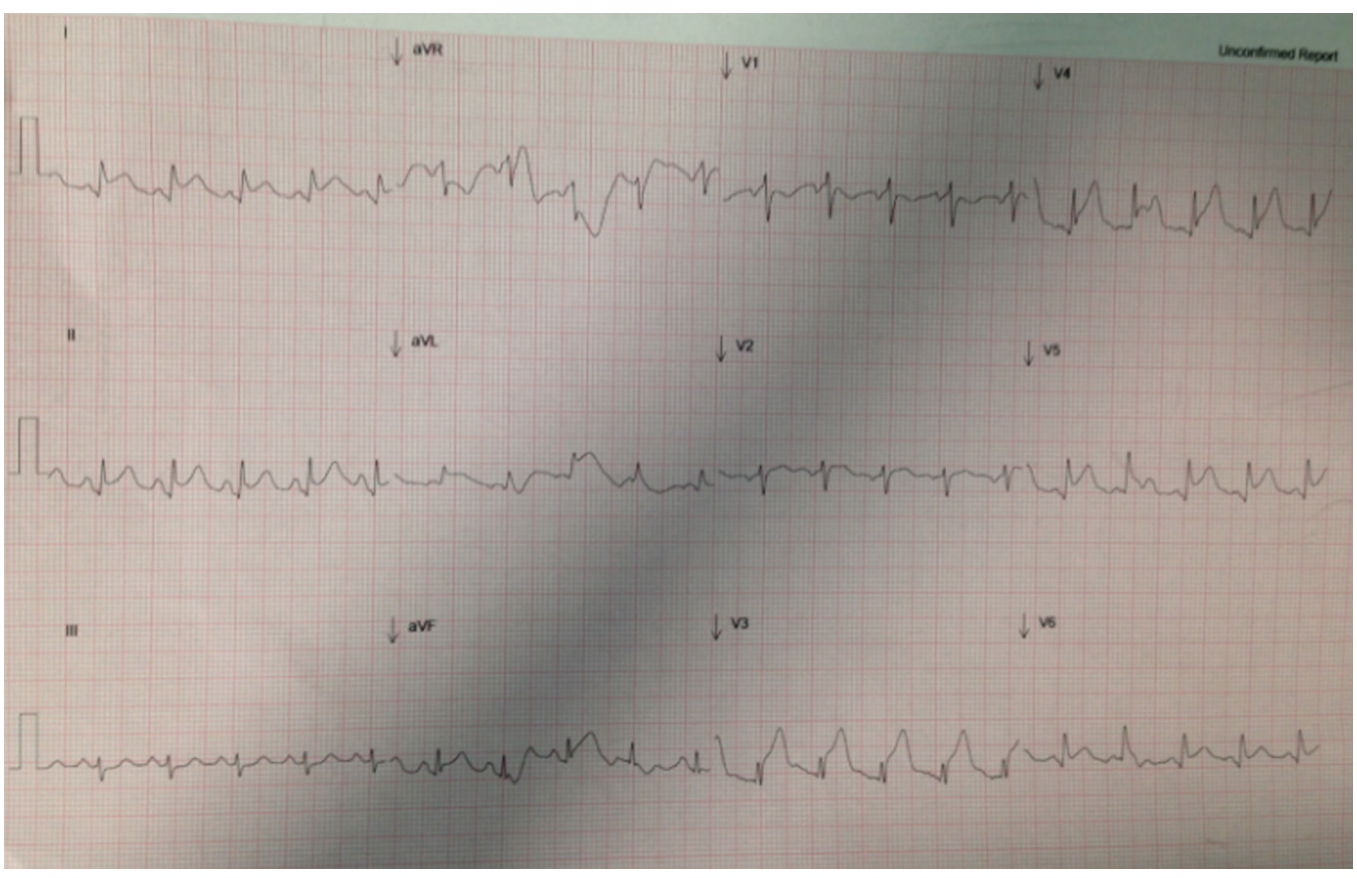

Figure 1. Post-induction ECG.

functional deterioration.

Her baseline bloods confirmed a hemoglobin of 9.1, with normal clotting and a normal renal function (urea 4.4, creatinine 73).

The patient underwent a gentle induction with fentanyl, propofol, and atracurium. Three minutes later, she developed bigeminy, with a stable blood pressure but oxygen requirements increasing to $80 \%$. Ten minutes post induction, ECG showed sinus rhythm with elevated ST segments in leads I, II, V3-6 (Fig. 1).

The procedure was abandoned and aspirin and clopidogrel were administered.

After discussion with the cardiology team, the patient was

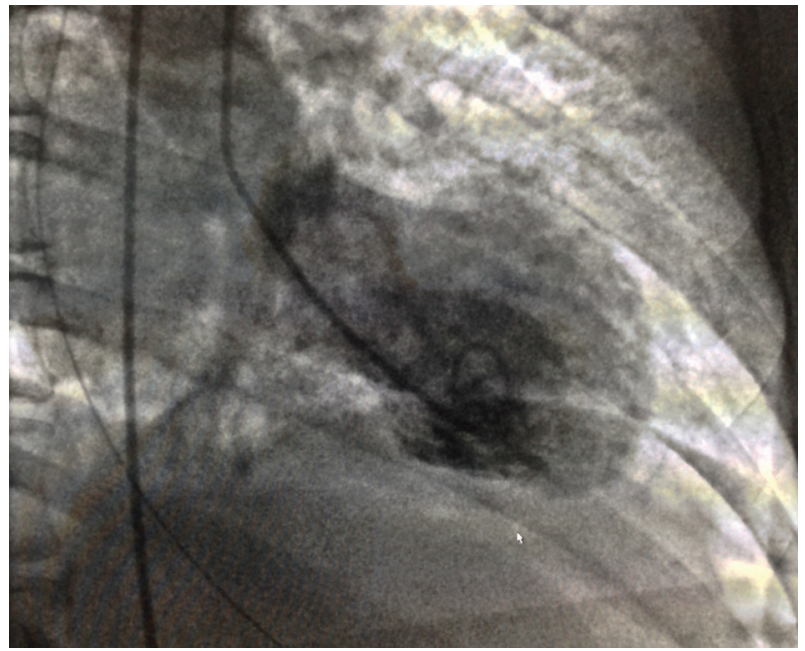

Figure 2. Ventriculogram. transferred to the cardiac catheterization laboratory with suspected myocardial infarction.

Subsequent angiogram demonstrated mild arterial disease but no occlusion and her post-procedure echocardiogram showed an ejection fraction of $20 \%$ with apical dilatation in keeping with Takotsubo cardiomyopathy. This was confirmed on contrast left ventriculogram (Fig. 2). During this period, she maintained a good blood pressure throughout.

Her troponin peaked at $8 \mathrm{~h}$ at 1,287 with a $12 \mathrm{~h}$ level of 1,026. ST segment started to resolve within the first $12 \mathrm{~h}$ and had reverted by the following day.

Initially post procedure, she remained intubated and ventilated awaiting transfer planning, prior to which she was extubated and moved to the high dependency unit. Later that evening, a portable chest X-ray confirmed the development of pulmonary edema which was managed with continuous positive pressure ventilation (CPAP) and she became hypotensive requiring noradrenaline and dobutamine infusions. Treatment during her high dependency admission was supportive and the higher-level organ support was weaned on the second day. She made a good recovery by day 3 , requiring low flow oxygen and weaned off all cardiovascular support.

\section{Discussion}

Takotsubo cardiomyopathy is associated with acute physical or emotional stress, the likely pathophysiological mechanism being a surge in endogenous catecholamines.

Our patient was electively admitted, had undergone anesthesia previously without complications and was used to the hospital environment with her previous and recent admissions for COPD, so although emotional stress is a well recognized 
cause for Takotsubo, it is interesting that this occurred post induction, with the secession of emotional stress due to post induced unconsciousness with anesthesia and prior to any procedure.

It is difficult to determine precisely the etiology and sequence of pathological events in this case, the patient had a normal pre-operative ECG and pre-induction trace on the monitor leading us to believe an iatrogenic stress related to medical procedures or the anesthetic agents themselves may have been the cause. However the anesthetic agents chosen in this case for induction are traditionally associated with a smoother induction with lower catecholamine release. The patient was given fentanyl $100 \mu \mathrm{g}$ to obtund the stimulating effects of laryngoscope and a small dose of propofol $50 \mathrm{mg}$ with top ups of $20 \mathrm{mg}$ and $20 \mathrm{mg}$ to good physiological effect. Following this she received a bolus of atracurium prior to laryngoscopy. She was a grade one intubation and as such received minimal stimulation. She had a good blood pressure and heart rate prior to induction, with no requirement for boluses of sympathomimetics post induction. She maintained a good mean arterial pressure throughout, a response to catecholamines. The inhalation agent sevoflurane traditionally is less irritant than other agents.

She was followed up with an echo at 6 weeks which proved resolution of her ejection fraction to $56 \%$ and the diagnosis of Takotsubos was confirmed.

\section{References}

1. Kodama K, Haze K, Hori M, editors. Clinical Aspect of Myocardial Injury: from Ischemia to Heart Failure. Tokyo: Kagakuhyoronsha Publishing Co.; 1990. p. 56-64.

2. Klinceva M, Widimsky P, Pesl L, Stasek J, Tousek F, Vambera M, Bilkova D. Prevalence of stress-induced myocardial stunning (Tako-Tsubo cardiomyopathy) among patients undergoing emergency coronary angiography for suspected acute myocardial infarction. Int J Cardiol. 2007;120(3):411-413.

3. Pilliere R, Mansencal N, Digne F, Lacombe P, Joseph T, Dubourg O. Prevalence of tako-tsubo syndrome in a large urban agglomeration. Am J Cardiol. 2006;98(5):662-665.

4. Liu S, Dhamee MS. Perioperative transient left ventricular apical ballooning syndrome: Takotsubo cardiomyopathy: a review. J Clin Anesth. 2010;22(1):64-70.

5. Littlejohn FC, Syed O, Ornstein E, Connolly ES, Heyer EJ. Takotsubo cardiomyopathy associated with anesthesia: three case reports. Cases J. 2008;1(1):227. 